\title{
Estudio prospectivo comparativo del manejo de la analgesia preventiva en pacientes sometidos a artroplastia total de rodilla primaria
}

\author{
Fernando A. Lopreite, Santiago Iglesias, Daniel Veloz Serrano, Alfredo Montero Vinces, Hernán del Sel \\ Servicio de Ortopedia y Traumatología, Hospital Británico de Buenos Aires, Ciudad Autónoma de Buenos Aires, Argentina
}

\begin{abstract}
RESUMEN
Introducción: La analgesia preventiva es aquella que se administra horas o días antes de una artroplastia total de rodilla con el fin de reducir el dolor posoperatorio. El objetivo de este estudio fue comparar y analizar la eficacia clínica de la analgesia preventiva en los pacientes sometidos a un reemplazo total de rodilla. Materiales y Métodos: Noventa y seis pacientes, divididos en dos grupos de 48 pacientes, fueron evaluados prospectivamente y comparados entre noviembre de 2018 y marzo de 2019 . El grupo A recibió analgesia preoperatoria con una combinación de etoricoxib, tramadol y paracetamol, por vía oral, 2 horas antes de la cirugía. El grupo B de control no recibió analgesia. El dolor posoperatorio de los pacientes se evaluó de acuerdo con sus propios registros en una escala analógica visual a las $12,24,48,72 \mathrm{~h}$ y una semana después de la cirugía. Resultados: Se observó una diferencia significativa a las $24 \mathrm{~h}$ entre ambos grupos, el dolor fue significativamente menor en el grupo $\mathrm{A}(\mathrm{p}=0,001)$, a las $48 \mathrm{~h}$ la diferencia fue estadísticamente menor que a las $24 \mathrm{~h}(\mathrm{p}=0,016)$. También se observó que el grupo de control requirió más cantidad de rescates, aunque la diferencia solo fue significativa a las 24 h ( $p=0,047)$. Conclusión: La combinación de etoricoxib, tramadol y paracetamol es eficaz, segura, económica y fácil de administrar, y la tasa de efectos adversos de la analgesia es baja; por lo tanto, representa un esquema adecuado para el manejo de la analgesia preventiva.
\end{abstract}

Palabras clave: Artroplastia de rodilla; dolor; analgesia preventiva.

Nivel de Evidencia: Ilb

Comparative Prospective Study of the Management of Preventive Analgesia in Patients Undergoing Primary Total Knee Arthroplasty

\section{ABSTRACT}

Background and Objective: Preventive analgesia is one that is administered hours or days before a total knee arthroplasty in order to reduce postoperative pain. The objective of this study was to compare and analyze the clinical efficacy of the use of preventive analgesia in patients undergoing total knee replacement. Materials and Methods: Ninety-six patients, divided into two groups of 48 patients, were evaluated prospectively and comparatively between November 2018 and March 2019. Group A received preoperative analgesia with a combination of etoricoxib, tramadol, and paracetamol, orally, 2 hours before surgery. Control group $\mathrm{B}$, did not receive anything. The postoperative pain of the patients was evaluated according to their own records on a visual analog scale at 12, 24, 48, 72 hours and one week after surgery. Results: A significant difference was observed at 24 hours between both groups, pain was significantly less for group A $(p=0.001)$, at 48 hours the difference was statistically lower than at 24 hours $(p=0.016)$. It was also observed that the control group required a greater number of rescues, although the difference was only significant at 24 hours $(p=0.047)$. Conclusion: The combination of etoricoxib, tramadol and paracetamol represents an effective, safe, economical, easy to administer form with a low rate of analgesia adverse effects; which represents an adequate scheme for the management of preventive analgesia.

Key words: Knee arthrosplasty; pain; preemptive analgesia.

Level of Evidence: Ilb

Recibido el 21-5-2020. Aceptado luego de la evaluación el 5-7-2020 • Dr. FERNANDO A. LOPREITE • fernandolopreite@ hotmail.com

https://orcid.org/0000-0002-2065-8649

Cómo citar este artículo: Lopreite FA, Iglesias S, Veloz Serrano D, Montero Vinces A, del Sel H. Estudio prospectivo comparativo del manejo de la analgesia preventiva en pacientes sometidos a artroplastia total de rodilla primaria. Rev Asoc Argent Ortop Traumatol 2021;86(1):31-38. https://doi.org/10.15417/issn. 1852-7434.2021.86.1.1125 


\section{INTRODUCCIÓN}

La artroplastia total es el procedimiento por excelencia utilizado para tratar la patología degenerativa de la rodilla. En la segunda mitad del siglo pasado, esta cirugía fue considerada uno de los avances quirúrgicos más relevantes. En la Argentina, se ha convertido en uno de los procedimientos de cirugía ortopédica más realizados. Sin embargo, hasta un 30\% de los pacientes sometidos a este procedimiento no refiere mejorías importantes en la calidad de vida luego del año de la operación. El dolor posoperatorio referido por los pacientes es uno de los principales problemas que tiene que resolver el cirujano. Estudios demuestran que aquellos pacientes que, antes de la cirugía, sufrían niveles elevados de dolor, luego de ellas, tendrán peores resultados y estarán insatisfechos con la intervención. Esto, muchas veces, trae aparejado una estancia hospitalaria prolongada, una rehabilitación posoperatoria tórpida y una alteración en relación médico-paciente. ${ }^{1}$

Múltiples son los métodos utilizados para prevenir y tratar el dolor posoperatorio: anestesia raquídea, bloqueo de nervios periféricos, infiltración articular, opioides, antinflamatorios no esteroides (AINE) y corticoides durante el posoperatorio. ${ }^{2-4}$

Podemos definir a la analgesia preventiva como la que se administra horas o días antes de una cirugía con el objetivo de disminuir el dolor perioperatorio.

El uso de analgésicos inmediatamente antes de la intervención para aliviar el dolor posoperatorio y los requerimientos analgésicos posoperatorios tiene un fundamento claro: disminuir el dolor agudo ante la lesión, prevenir la modulación patológica relacionada con el dolor sobre el sistema nervioso central, prevenir el consumo de analgésicos e inhibir la persistencia del dolor posoperatorio y evitar el dolor crónico. ${ }^{3-5}$

Estudios en animales confirman que la administración de analgésicos antes de una lesión tisular es más eficaz para controlar el dolor que si se administran después; sin embargo, su eficacia clínica real es muy controvertida. ${ }^{6}$

Algunos ensayos clínicos muestran que el momento de comenzar la analgesia no afecta el control del dolor posoperatorio, cualquiera sea el método de analgesia empleado. No obstante, esta conclusión puede ser errónea debido a que estos ensayos se basan en la evaluación del dolor a corto plazo y es posible que existan diferencias según el tipo de cirugía. ${ }^{7,8}$

El objetivo de este estudio fue comparar y analizar la eficacia clínica de la analgesia preventiva en los pacientes sometidos a reemplazo total de rodilla y su impacto en la administración de analgesia posoperatoria, la rehabilitación y los días de internación hospitalaria.

\section{MATERIALES Y MÉTODOS}

Este estudio se llevó a cabo en el Hospital Británico, ubicado en la Ciudad Autónoma de Buenos Aires. Se trató de un protocolo prospectivo. Se evaluó a 96 pacientes seleccionados en forma aleatorizada y tratados por dos cirujanos de nuestra institución, entre noviembre de 2018 y marzo de 2019.

La recolección de datos y su evaluación estuvieron a cargo de V. D. e I. S., médicos del mismo Servicio, quienes fueron independientes de los cirujanos que trataron a los pacientes de este estudio.

Se formaron dos grupos de 48 pacientes: el grupo A al que se le indicó un plan de analgesia antes de la cirugía y el grupo B de control.

Se incluyó a pacientes con gonalgia de moderada a intensa unilateral que sufrían enfermedad degenerativa crónica de la rodilla (clasificación de Ahlback $\geq 3$ ) que tuviera indicación de reemplazo total de rodilla. Se excluyó a quienes presentaban: 1) enfermedad ya conocida o alergia que contraindicara alguno de los fármacos del protocolo, 2) cirugías de revisión de prótesis, 3) antecedentes psiquiátricos, 4) gonalgia bilateral o 5) dolor crónico de otra articulación.

Ambos grupos recibieron el mismo tipo de anestesia (bloqueo raquídeo y bloqueo del canal safeno), así como también el mismo plan de analgesia posoperatoria. En todos los casos, se realizó un abordaje pararrotuliano interno y no se colocó hemosuctor. En 20 pacientes del grupo A y cinco del grupo B, se usó manguito hemostático durante la cirugía, el cual estuvo supeditado a la decisión del médico tratante considerando los antecedentes del paciente o a una indicación quirúrgica.

El plan de analgesia posoperatoria durante los dos primeros días fue el mismo para los dos grupos:

1) dos ampollas de morfina diluida en $500 \mathrm{ml}$ de solución fisiológica, por vía intravenosa, en goteo de $21 \mathrm{ml} / \mathrm{h}$,

2) diclofenac $75 \mathrm{mg}$, por vía intravenosa, c/12 h,

3) una ampolla de morfina de rescate, por vía intravenosa, según necesidad. 
Luego de las 48 h, el plan analgésico se rotó a la vía oral, de la siguiente manera (se continuó con el mismo esquema una vez dado de alta):

1) diclofenac $75 \mathrm{mg} \mathrm{c} / 12 \mathrm{~h}$,

2) tramadol $100 \mathrm{mg}$ de rescate, según necesidad (hasta $100 \mathrm{mg} \mathrm{c/8} \mathrm{h).}$

Todos recibieron omeprazol en ayunas como protección gástrica, metoclopramida como rescate ante un episodio de náuseas o vómitos y profilaxis antitrombosis venosa profunda diaria con enoxaparina hasta los 21 días de la operación (40 mg, por vía subcutánea).

La rehabilitación comenzó inmediatamente después de la cirugía con ejercicios isométricos en cama, bipedestación y dembulación con asistencia (andador o bastones canadienses) a las 12-24 h de acuerdo con la posibilidad del paciente según el dolor. Se les solicitó a los pacientes que completaran una ficha para registrar el dolor según una escala analógica visual a las $12,24,48,72 \mathrm{~h}$ y a la semana poscirugía, así como la cantidad de rescates de opioides utilizados luego de ser dados de alta. Asimismo, se los interrogó sobre el grado de satisfacción con el médico tratante, los efectos adversos de la medicación indicada y su rehabilitación.

Se recolectaron datos de la hoja de enfermería y la historia clínica acerca de: rescates de opioides durante la estancia hospitalaria, efectos adversos de la analgesia y tiempo de internación.

Con los datos recolectados se llevó a cabo el análisis estadístico mediante la prueba estadística de U de MannWhitney para dos muestras.

\section{Protocolo de analgesia preventiva}

Todos los pacientes fueron citados $48 \mathrm{~h}$ antes de la cirugía para darles las indicaciones prequirúrgicas. Solo a los del grupo A se les indicó el siguiente plan de analgesia preventiva por vía oral:

1) paracetamol $1 \mathrm{~g}$,

2) etoricoxib $90 \mathrm{mg}$,

3) tramadol $100 \mathrm{mg}$ en comprimidos.

A los pacientes de este grupo se les pidió que tomaran la medicación con un sorbo de agua, 2 h antes de la cirugía.

El equipo de anestesia les aplicó, como rutina, un corticoide a los pacientes de ambos grupos en la inducción anestésica.

\section{RESULTADOS}

Todos los datos fueron recolectados y evaluados por dos de los autores. El grupo A tenía una media de la edad de 73.4 años (rango 40-89) y el grupo B (control), de 72.5 años (rango 42-87).

Se operaron 20 rodillas derechas y 28 rodillas izquierdas en el grupo A y 29 rodillas derechas y 19 rodillas izquierdas en el grupo B.

Doce de las 48 rodillas del grupo A tenían un deseje en valgo y 36, en varo; en el grupo B, 10 eran rodillas valgas y 38 , rodillas varas.

En el grupo A, estaba conformado por 30 mujeres y 18 hombres, y el grupo B, por 27 mujeres y 21 hombres.

Según los datos analizados de la escala analógica visual (del 0 al 10) administrada por los pacientes, se puede observar que: (Figura 1)

1) A las $12 \mathrm{~h}$ de concluir la cirugía, la diferencia entre los dos grupos fue estadísticamente insignificante $(\mathrm{p}=$ $0,716)$.

2) La mayor diferencia entre el grupo A y el grupo B se observó a las 24 h de la cirugía. El dolor fue significativamente menor para el grupo A $(\mathrm{p}=0,008)$.

3) A las $48 \mathrm{~h}$, la diferencia fue estadísticamente menor que a las $24 \mathrm{~h}$, se mantuvo estadísticamente significativa $(\mathrm{p}=0,019)$.

Al evaluar la cantidad de rescates de morfina, se observó que el grupo de control requirió más cantidad de rescates, aunque la diferencia solo fue estadísticamente significativa a las $24 \mathrm{~h}$ ( $\mathrm{p}=0,083$ ) (Figura 2). 


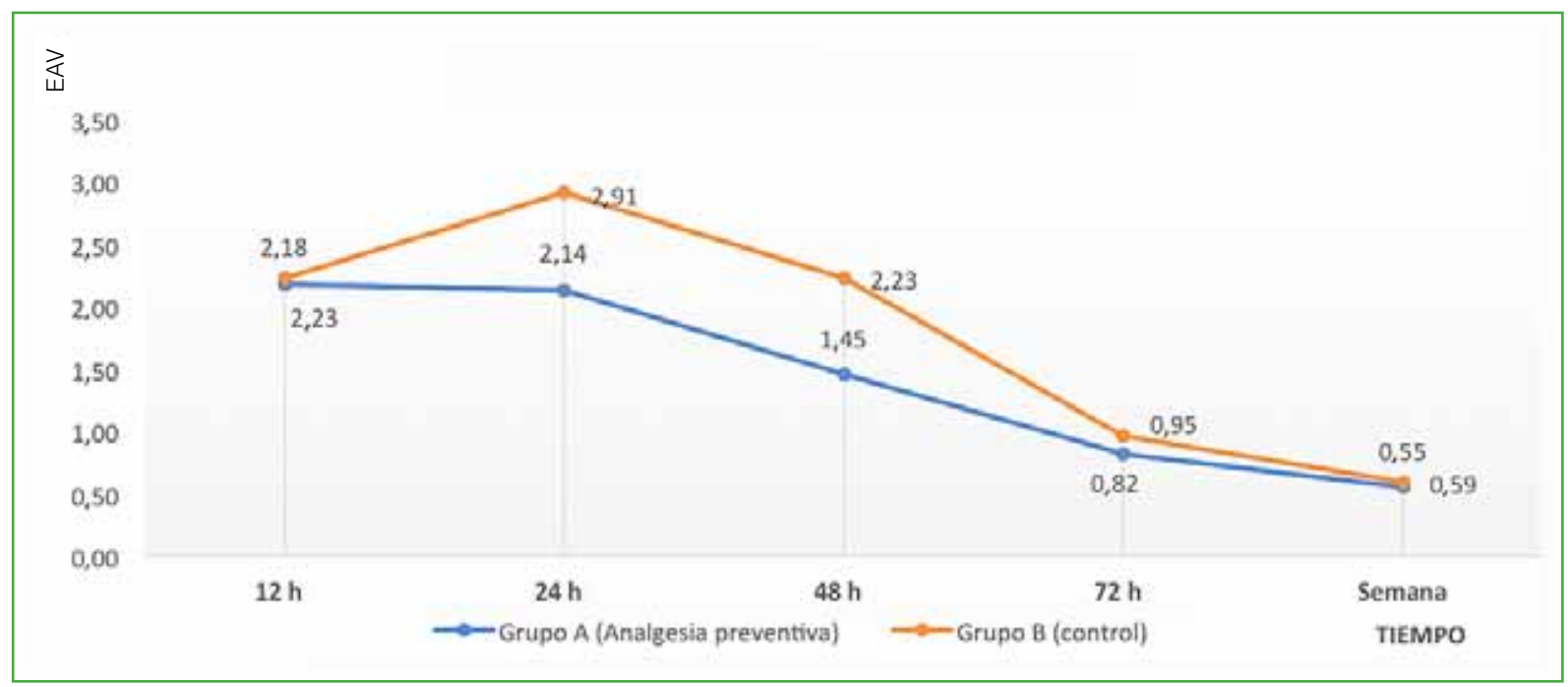

Figura 1. Escala analógica visual distribuida en el tiempo entre el grupo con analgesia preventiva y el grupo de control.

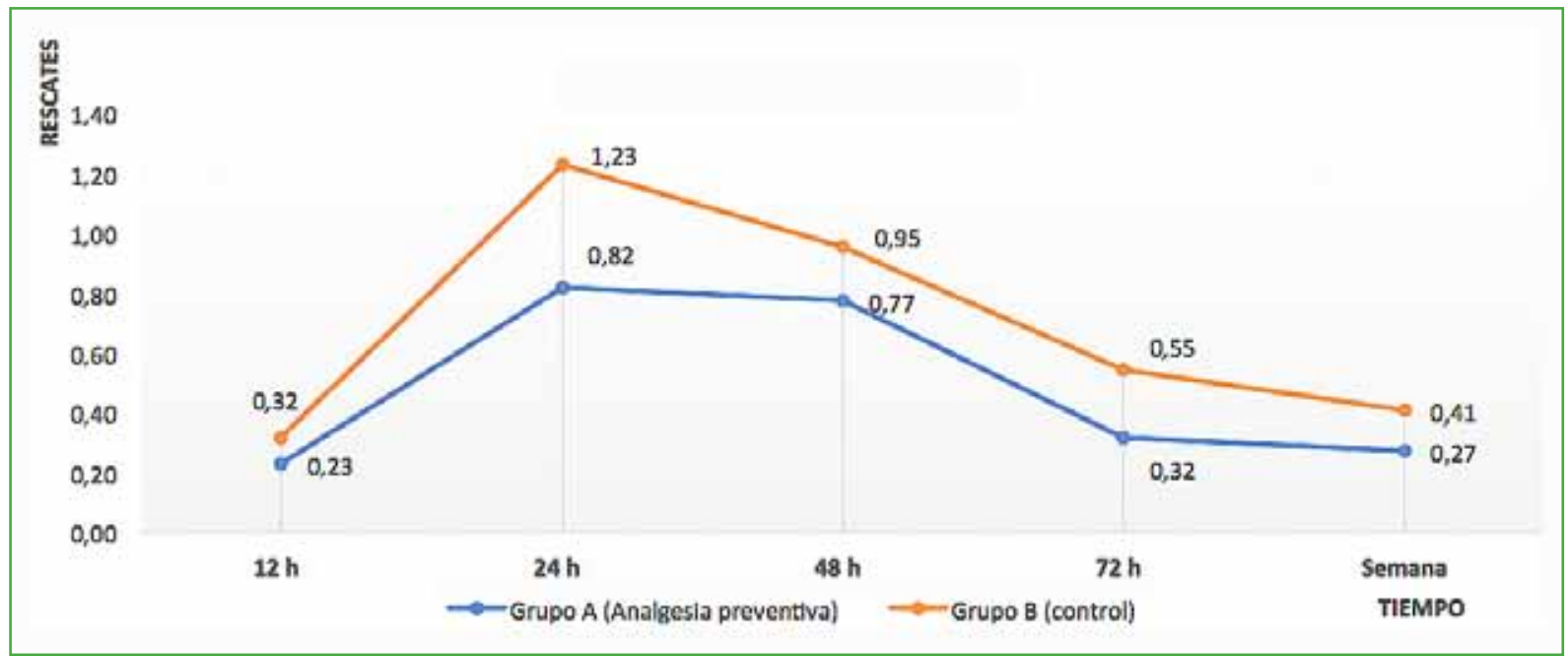

Figura 2. Administración de rescates en el tiempo.

Todos los pacientes del grupo A iniciaron su rehabilitación caminando antes de las 24 horas. Mientras que el grupo B no inició la marcha hasta las 48 h, solo realizaba sedestación y ejercicios isométricos a las 24 h. Veinte refirieron que había sido por el dolor posoperatorio. Por lo tanto, los pacientes del grupo A pudieron iniciar antes la rehabilitación intrahospitalaria y disminuir los días de internación (Figura 3). Para el grupo A los días fueron, en promedio, 3.68 y, para el grupo B, 4.029 ( $\mathrm{p}=0,055)$, lo cual resultó estadísticamente significativo.

También se interrogó a los pacientes sobre su grado de satisfacción con su médico de cabecera luego de la cirugía, en una escala del 1 al 5 ( 1 = poco satisfecho; 5 = muy satisfecho). El promedio para el grupo A fue de 4,68 puntos y, para el grupo B, de 4,52 puntos, lo que resultó estadísticamente insignificante $(\mathrm{p}=0,103)$.

Las complicaciones fueron las siguientes: de los 96 pacientes evaluados, 20 sufrieron complicaciones menores: nueve (grupo A: 6, grupo B: 3) con complicaciones gastrointestinales concomitantes (diarrea, náuseas, vómitos), cuatro (grupo A: 2, grupo B: 2) tuvieron fiebre en el posoperatorio inmediato ( $>38^{\circ}$, un episodio aislado), tres (grupo A: 2, grupo B: 1) presentaron débito abundante prolongado por la herida ( $>3$ días) sin complicaciones 
aparejadas, fueron dados de alta con el vendaje seco luego de un control por más de $48 \mathrm{~h}$ a partir del último vendaje manchado; dos pacientes del grupo A sufrieron cefalea intensa y dos del grupo B presentaron un episodio de desaturación que mejoró con oxígeno al $100 \%$ en la sala general sin más complicaciones.

Entre las complicaciones mayores, un paciente del grupo A y uno del grupo B sufrieron una trombosis venosa profunda en el posoperatorio inmediato que requirió tratamiento médico, se los mantuvo internados hasta obtener valores del coagulograma adecuados y un paciente del grupo B sufrió una infección aguda de la prótesis que requirió una limpieza quirúrgica y tratamiento con antibióticos, sin necesidad de retirar la prótesis.

Ninguna de las complicaciones tuvo relación directa con la medicación administrada.

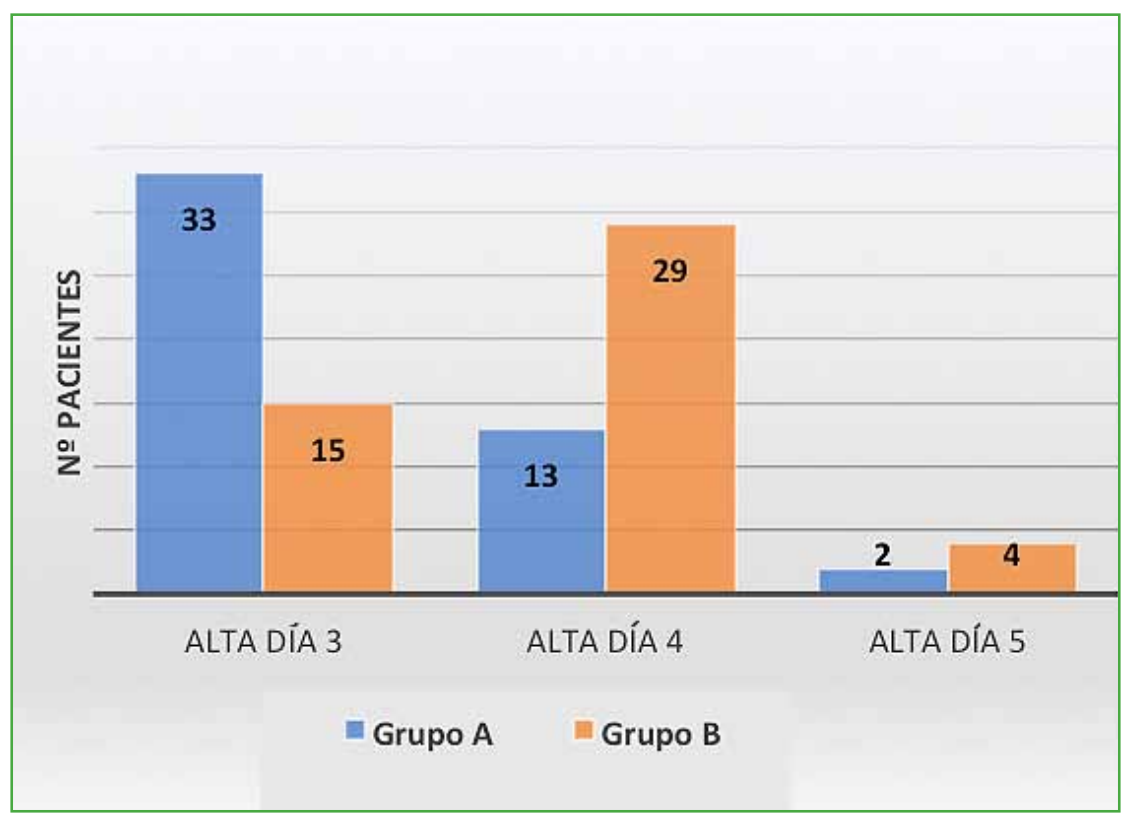

Figura 3. Distribución de los pacientes según el día de alta, por grupo.

\section{DISCUSIÓN}

La artroplastia total de rodilla produce un dolor de moderado a intenso en el primer día posoperatorio, en el 75\% de los pacientes y después de dos semanas en el $30-40 \%$ de los casos. ${ }^{9}$ Esto puede tener implicancias, no solo por el malestar que puede generar, sino también por alterar el proceso habitual de rehabilitación, la rigidez articular, el dolor residual crónico y el malestar en la relación médico-paciente. ${ }^{10}$

Los AINE, tanto los inhibidores de la Cox-1 como los de la Cox-2, administrados antes de la cirugía, han sido eficaces para controlar el dolor. Un metanálisis de Ong y cols. reveló que los AINE, en múltiples tipos de cirugías, solos o combinados, disminuyen el consumo de analgésicos en el posoperatorio y el tiempo hasta la primera solicitud de rescate de opioide. ${ }^{11}$ En otro estudio de Alexander y cols., se demostró que la administración preoperatoria de $75 \mathrm{mg}$ de diclofenac por vía intravenosa o $60 \mathrm{mg}$ de ketorolac antes del reemplazo total de rodilla reduce significativamente los requerimientos de morfina y los efectos secundarios asociados a esta. ${ }^{12}$ En un estudio de 11 pacientes llevado a cabo por Renner y cols., se comprobó que la administración de etoricoxib $2 \mathrm{~h}$ antes de la cirugía permite alcanzar una concentración efectiva del fármaco en tejidos críticos, una menor producción de mediadores proinflamatorios y un mejor control del dolor en el posoperatorio inmediato, si bien fue realizado en pacientes sometidos a un reemplazo articular de cadera, creemos que es trasladable al reemplazo total de rodilla. ${ }^{13}$ En nuestro estudio, preferimos administrar este último AINE, debido a que los inhibidores de la Cox-2 tienen la ventaja de no producir alteraciones de la agregación plaquetaria, lo que evita las complicaciones hemorrágicas y provoca menos efectos adversos gastrointestinales. 
Los opioides también son eficaces como analgesia preventiva. Hendolin y cols. realizaron un estudio con 41 pacientes a quienes administraron $0,14 \mathrm{mg} / \mathrm{kg}$ de morfina, por vía intramuscular, una hora antes del reemplazo total de rodilla. Esto alivió el dolor en el posoperatorio inmediato. ${ }^{14}$ En nuestro estudio, optamos por el tramadol, ya que, por su posología y su presentación en comprimidos de $100 \mathrm{mg}$, es fácil de administrar.

No hallamos estudios publicados sobre la administración de paracetamol, de forma aislada, para prevenir el dolor; sí tuvo un efecto beneficioso al ser indicado en combinación con otros fármacos. En un estudio de Skinner y Shintani, se observó que el paracetamol administrado con rofecoxib, tramadol y dexametasona mejoraba significativamente el dolor en el posoperatorio. ${ }^{15}$

En nuestro estudio, combinando los tres fármacos, etoricoxib $90 \mathrm{mg}$, paracetamol $1 \mathrm{~g}$ y tramadol $100 \mathrm{mg}$, por vía oral, $2 \mathrm{~h}$ antes de la cirugía, se lograron mejores resultados en el manejo del dolor posoperatorio en las primeras $24 \mathrm{~h}$ y hubo una menor solicitud de rescates de morfina. Esto se traduce en comodidad para el paciente, una rehabilitación más rápida y una estancia hospitalaria más corta.

Otros grupos de fármacos indicados para el dolor han sido útiles, como los corticoides, la dexametasona y la metilprednisolona serían beneficiosos administrados horas antes de la cirugía de reemplazo articular. ${ }^{14,16,17}$ Estos medicamentos son aplicados de rutina por el equipo de anestesia, durante la inducción, para disminuir el efecto inflamatorio de la cirugía.

La bibliografía es contundente acerca del beneficio de la analgesia multimodal para el tratamiento preventivo del dolor, encontramos múltiples estudios que lo demuestran. ${ }^{15,18-21}$ Podemos definir a la analgesia multimodal como aquella que se logra combinando diferentes analgésicos que actúan por diferentes mecanismos y en diferentes sitios de la transmisión del dolor con el fin de potenciar la analgesia y disminuir los efectos colaterales de los medicamentos utilizados, al requerir dosis menores de estos. ${ }^{22}$

Las estrategias para reducir, al mínimo, el dolor en el posoperatorio son múltiples; sin embargo, quedan supeditadas a la propia experiencia del médico tratante, ya que no existen estudios con la suficiente evidencia, ni guías clínicas que determinen que un protocolo de analgesia sea mejor que otro para prevenir el dolor luego de una cirugía de reemplazo articular de rodilla. No obstante, hay metanálisis y consensos elaborados por expertos que proponen algunas pautas y recomendaciones para el manejo de la analgesia preventiva. ${ }^{5,23}$

Un tema que trae controversias es el uso del manguito hemostático. En un estudio publicado por Liu y cols., se demostró que los pacientes a los que no se le colocaba torniquete tenían menos dolor en el posoperatorio temprano que los del grupo con torniquete. ${ }^{24}$ Sin embargo, en otro estudio de Kim y cols., no se encontró significancia en el uso de torniquete con relación al dolor. ${ }^{25}$ En nuestro estudio, si bien el grupo A tenía 15 pacientes más que el grupo de control a los cuales se les indicó el manguito hemostático, aun así, sintieron menos dolor que los del grupo B.

Las limitaciones de nuestro estudio son: la baja cantidad de pacientes, la falta de seguimiento a largo plazo para evaluar el dolor y la rehabilitación a la semana de la cirugía, una muestra con diagnósticos heterogéneos (se incluyó a todos los pacientes con rodillas dolorosas degenerativas). Como fortalezas destacamos: el diseño prospectivo y aleatorizado, y ser un estudio comparativo entre dos poblaciones similares.

\section{CONCLUSIONES}

Creemos que nuestro estudio arrojó resultados representativos y comparables con la bibliografía consultada. El protocolo de analgesia administrado antes de la cirugía (etoricoxib, tramadol y paracetamol) logró una mejoría significativa en el manejo del dolor posoperatorio y esto significó mejorías en la rehabilitación y disminuyó los días de internación. A su vez, representa una combinación segura, económica, fácil de administrar y con baja tasa de efectos adversos, lo cual demuestra ser una buena combinación de fármacos.

Es importante destacar que la analgesia preventiva no es una herramienta aislada, sino que requiere la combinación de una correcta anestesia y un plan de analgesia posoperatoria adecuado para lograr una mejora significativa en el bienestar del paciente y lo que esto trae aparejado.

Conflicto de intereses: Los autores no declaran conflictos de intereses. 


\section{BIBLIOGRAFÍA}

1. Serra-Sutton V, Allepuz A, Martínez O, Espallargues M. Factores relacionados con la calidad de vida al año de la artroplastia total de cadera y rodilla: Estudio multicéntrico en Cataluña. Rev Esp Cir Ortop Traumatol 2013;57(4):254-62. https://doi.org/10.1016/j.recot.2013.04.002

2. Badner NH, Bourne RB, Rorabeck CH, MacDonald SJ, Doyle JA. Intra-articular injection of bupivacaine in knee-replacement operations. Results of use for analgesia and for preemptive blockade. J Bone Joint Surg Am 1996;78(5):734-8. https://doi.org/10.2106/00004623-199605000-00013

3. Browne C, Copp S, Reden L, Pulido P, Colwell C. Bupivacaine bolus injection versus placebo for pain management following total knee arthroplasty. J Arthroplasty 2004;19(3):377-80. https://doi.org/10.1016/j.arth.2003.10.012

4. Fowler SJ, Symons J, Sabato S, Myles PS. Epidural analgesia compared with peripheral nerve blockade after major knee surgery: A systematic review and meta-analysis of randomized trials. Br J Anaesth 2008;100(2):154-64. https://doi.org/10.1093/bja/aem373

5. Díaz-Heredia J, Loza E, Cebreiro I, Ruiz Iban M. Analgesia preventiva en artroplastia de cadera o rodilla: Una revisión sistemática. Rev Esp Cir Ortop Traumatol 2015;59(2):73-90. https://doi.org/10.1016/j.recot.2014.09.004

6. Katz J, McCartney CJL. Current status of pre-emptive analgesia. Curr Opin Anaesthesiol 2002;15(4):435-41. https://doi.org/10.1097/00001503-200208000-00005.9

7. McCarthy GC, Megalla SA, Habib AS. Impact of intravenous lidocaine infusion on postoperative analgesia and recovery from surgery: A systematic review of randomized controlled trials. Drugs 2010;70(9):1149-63. https://doi.org/10.2165/10898560-000000000-00000

8. Gottschalk A, Raja SN. Severing the link between acute and chronic pain: The anesthesiologist's role in preventive medicine. Anesthesiology 2004;101(5):1063-5. https://doi.org/10.1097/00000542-200411000-00004

9. Andersen LØ, Gaarn-Larsen L, Kristensen BB, Husted H, Otte KS, Kehlet H. Subacute pain and function after fasttrack hip and knee arthroplasty. Anaesthesia 2009;64(5):508-13. https://doi.org/10.1111/j.1365-2044.2008.05831.x

10. Dorr LD, Chao L. The emotional state of the patient after total hip and knee arthroplasty. Clin Orthop Relat Res 2007;463:7-12. PMID: 17960669

11. Ong CKS, Lirk P, Seymour RA, Jenkins BJ. The efficacy of preemptive analgesia for acute postoperative pain management: A meta-analysis. Anesth Analg 2005;100(3):757-73. https://doi.org/10.1213/01.ANE.0000144428.98767.0E

12. Alexander R, El-Moalem HE, Gan TJ. Comparison of the morphine-sparing effects of diclofenac sodium and ketorolac tromethamine after major orthopedic surgery. J Clin Anesth 2002;14(3):187-92. https://doi.org/10.1016/s0952-8180(01)00382-8

13. Renner B, Walter G, Strauss J, Fromm MF, Zacher J, Brune K. Preoperative administration of etoricoxib in patients undergoing hip replacement causes inhibition of inflammatory mediators and pain relief. Eur J Pain 2012;16(6):838-48. https://doi.org/10.1002/j.1532-2149.2011.00062.x

14. Hendolin H, Nuutinen L, Kokki H, Tuomisto L. Does morphine premedication influence the pain and consumption of postoperative analgesics after total knee arthroplasty? Acta Anaesthesiol Scand 1996;40(1):81-5. https://doi.org/10.1111/j.1399-6576.1996.tb04391.x

15. Skinner HB, Shintani EY. Results of a multimodal analgesic trial involving patients with total hip or total knee arthroplasty. Am J Orthop (Belle Mead NJ) 2004;33(2):85-92; discussion 92.18. PMID: 15005598

16. Shahraki AD, Feizi A, Jabalameli M, Nouri S. The effect of intravenous dexamethasone on post-cesarean section pain and vital signs: A double-blind randomized clinical trial. J Res Pharm Pract 2013;2(3):99-104. https://doi.org/10.4103/2279-042X.12237019

17. Buvanendran A, Kroin JS, Della Valle CJ, Kari M, Moric M, Tuman KJ. Perioperative oral pregabalin reduces chronic pain after total knee arthroplasty: A prospective, randomized, controlled trial. Anesth Analg 2010;110(1):199-207. https://doi.org/10.1213/ANE.0b013e3181c4273a

18. Beaupre LA, Johnston DBC, Dieleman S, Tsui B. Impact of a preemptive multimodal analgesia plus femoral nerve blockade protocol on rehabilitation, hospital length of stay, and postoperative analgesia after primary total knee arthroplasty: A controlled clinical pilot study. Sci World J 2012: 273821. https://doi.org/10.1100/2012/273821

19. Eggers KA, Jenkins BJ, Power I. Effect of oral and i.v. tenoxicam in postoperative pain after total knee replacement. Br J Anaesth 1999;83(6):876-881. https://doi.org/10.1093/bja/83.6.876

20. Conselho Editorial CE. Editorial. Bol Goiano Geogr [Internet]. 2011;30(2). Disponible en: http://revistas.ufg.br/index.php/bgg/article/view/13785 
21. Rasmussen ML, Mathiesen O, Dierking G, Christensen BV, Hilsted KL, Larsen TK, et al. Multimodal analgesia with gabapentin, ketamine and dexamethasone in combination with paracetamol and ketorolac after hip arthroplasty: A preliminary study. Eur J Anaesthesiol 2010;27(4):324-30. https://doi.org/10.1097/EJA.0b013e328331c71d

22. Buvanendran A, Kroin JS. Multimodal analgesia for controlling acute postoperative pain. Curr Opin Anaesthesiol 2009;22(5):588-93. https://doi.org/10.1097/ACO.0b013e328330373a

23. Ruiz Ibán MA, Maculé F, Torner P, Gil Garay E, Oteo-Álvaro A, López Millán JM, et al. Consenso SECOTGEDOS sobre el control del dolor prequirúrgico en artrosis de rodilla y cadera. Rev Esp Cir Ortop Traumatol 2015;59(3):186-99. https://doi.org/10.1016/j.recot.2014.09.005

24. Liu D, Graham D, Gillies K, Gillies RM. Effects of tourniquet use on quadriceps function and pain in total knee arthroplasty. Knee Surg Relat Res 2014;26(4):207-13. https://doi.org/10.5792/ksrr.2014.26.4.207

25. Kim TK, Bamne AB, Sim JA, Park JH, Na YG. Is lower tourniquet pressure during total knee arthroplasty effective? A prospective randomized controlled trial. BMC Musculoskelet Disord 2019;20(1):1-9. https://doi.org/10.1186/s12891-019-2636-7 